PROCEEDINGS OF THE

AMERICAN MATHEMATICAL SOCIETY

Volume 139, Number 8, August 2011, Pages 2777-2782

S 0002-9939(2010)10722-0

Article electronically published on December 28, 2010

\title{
SUBSPACES OF ALMOST DAUGAVET SPACES
}

\author{
SIMON LÜCKING
}

(Communicated by Nigel J. Kalton)

\begin{abstract}
We study the almost Daugavet property, a generalization of the Daugavet property. We analyze what kind of subspaces and sums of Banach spaces with the almost Daugavet property have this property as well. The main result of the paper is that if $Z$ is a closed subspace of a separable almost Daugavet space $X$ such that the quotient space $X / Z$ contains no copy of $\ell_{1}$, then $Z$ has the almost Daugavet property too.
\end{abstract}

\section{INTRODUCTION}

V. Kadets, V. Shepelska, and D. Werner characterized in [2] a class of separable Banach spaces in three very different ways.

Theorem 1.1. For a separable Banach space $X$ the following conditions are equivalent:

(i) $S_{X}$ does not admit a finite $\varepsilon$-net consisting of elements of $S_{X}$ for any $\varepsilon<2$.

(ii) There is a sequence $\left(e_{n}\right)_{n \in \mathbb{N}}$ in $B_{X}$ such that for every $x \in X$

$$
\lim _{n \rightarrow \infty}\left\|x+e_{n}\right\|=\|x\|+1 \text {. }
$$

(iii) There is a norming subspace $Y \subset X^{*}$ such that the equation

$$
\|\operatorname{Id}+T\|=1+\|T\|
$$

holds true for every rank-one operator $T: X \rightarrow X$ of the form $T=y^{*} \otimes x$, where $x \in X$ and $y^{*} \in Y$.

Recall that a subspace $Y \subset X^{*}$ is said to be norming if for every $x \in X$

$$
\sup _{y^{*} \in S_{Y}}\left|y^{*}(x)\right|=\|x\| \text {. }
$$

Condition (i) can be rephrased using the so-called thickness of a Banach space $X$ that was introduced by R. Whitley in $\left[5\right.$. We call a set $F$ an inner $\varepsilon$-net of $S_{X}$ if $F \subset S_{X}$ and for every $x \in S_{X}$, there is a $y \in F$ with $\|x-y\| \leq \varepsilon$. Then the thickness $T(X)$ of a Banach space $X$ is defined by

$$
T(X)=\inf \left\{\varepsilon>0 \text { : there exists a finite inner } \varepsilon \text {-net of } S_{X}\right\} \text {. }
$$

Essentially, the thickness is a kind of inner measure of non-compactness of the unit sphere $S_{X}$.

Received by the editors July 17, 2010.

2010 Mathematics Subject Classification. Primary 46B04.

Key words and phrases. Daugavet property.

(C)2010 American Mathematical Society Reverts to public domain 28 years from publication 
R. Whitley showed that $1 \leq T(X) \leq 2$ if $X$ is infinite dimensional, in particular that $T\left(l_{p}\right)=2^{1 / p}$ for $1 \leq p<\infty$ and $T(C(K))=2$ if $K$ has no isolated points.

So condition (i) means that $T(X)=2$.

Condition (ii) links the investigation to the theory of types that were used by J.-L. Krivine and B. Maurey in 4. A type on a separable Banach space is a function of the form

$$
\tau(x)=\lim _{n \rightarrow \infty}\left\|x+e_{n}\right\|
$$

for some bounded sequence $\left(e_{n}\right)_{n \in \mathbb{N}}$. In [4] a convolution of types is defined and by means of this convolution the notion of $\ell_{1}$-types is introduced. A special $\ell_{1}$-type is generated by a sequence $\left(e_{n}\right)_{n \in \mathbb{N}}$ satisfying

$$
\tau(x)=\lim _{n \rightarrow \infty}\left\|x+e_{n}\right\|=\|x\|+1 .
$$

We call a sequence $\left(e_{n}\right)_{n \in \mathbb{N}}$ in $B_{X}$ satisfying this equation a canonical $\ell_{1}$-type sequence. The following proposition shows us that a canonical $\ell_{1}$-type sequence (or a suitable subsequence) almost behaves like the canonical base of $\ell_{1}$.

Proposition 1.2. Let $X$ be a separable Banach space, let $E_{1} \subset E_{2} \subset E_{3} \subset \ldots$ be a sequence of finite-dimensional subspaces whose union is dense in $X$, and let $\left(\varepsilon_{n}\right)_{n \in \mathbb{N}}$ be a sequence of positive real numbers that converges monotonically towards zero. If $\left(e_{n}\right)_{n \in \mathbb{N}}$ is a canonical $\ell_{1}$-type sequence, then we can pass to a subsequence $\left(f_{n}\right)_{n \in \mathbb{N}}$ of $\left(e_{n}\right)_{n \in \mathbb{N}}$ such that for every $x \in E_{n}$ and $y=\sum_{k=n+1}^{M} \lambda_{k} f_{k}$,

$$
\|x+y\| \geq\left(1-\varepsilon_{n}\right)\|x\|+\sum_{k=n+1}^{M}\left(1-\varepsilon_{k-1}\right)\left|\lambda_{k}\right| .
$$

Proof. Fix a sequence of positive real numbers $\left(\delta_{n}\right)_{n \in \mathbb{N}}$ with $\prod_{k=n}^{\infty}\left(1-\delta_{k}\right) \geq 1-\varepsilon_{n}$ for all $n \in \mathbb{N}$. There is a subsequence $\left(f_{n}\right)_{n \in \mathbb{N}}$ of $\left(e_{n}\right)_{n \in \mathbb{N}}$ such that for every $x \in \operatorname{span}\left(E_{n} \cup\left\{f_{1}, \ldots, f_{n}\right\}\right)$ and $\lambda \in \mathbb{K}$

$$
\left\|x+\lambda f_{n+1}\right\| \geq\left(1-\delta_{n}\right)(\|x\|+|\lambda|) .
$$

Then we have for every $x \in E_{n}$ and $y=\sum_{k=n+1}^{M} \lambda_{k} f_{k}$ as a consequence of the choice of $\left(\delta_{n}\right)_{n \in \mathbb{N}}$,

$$
\begin{aligned}
\|x+y\|=\left\|x+\sum_{k=n+1}^{M} \lambda_{k} f_{k}\right\| & \geq\left(1-\delta_{M-1}\right)\left\|x+\sum_{k=n+1}^{M-1} \lambda_{k} f_{k}\right\|+\left(1-\delta_{M-1}\right)\left|\lambda_{M}\right| \\
& \geq \cdots \geq \prod_{k=n}^{M-1}\left(1-\delta_{k}\right)\|x\|+\sum_{k=n+1}^{M}\left(1-\delta_{k-1}\right)\left|\lambda_{k}\right| \\
& \geq\left(1-\varepsilon_{n}\right)\|x\|+\sum_{k=n+1}^{M}\left(1-\varepsilon_{k-1}\right)\left|\lambda_{k}\right| .
\end{aligned}
$$

Condition (iii) links the investigation to the theory of Banach spaces with the Daugavet property introduced by V. Kadets, R. Shvidkoy, G. Sirotkin, and D. Werner in [3. We say that a Banach space $X$ has the Daugavet property with respect to $Y$ if the Daugavet equation (1.1) holds true for every rank-one operator $T: X \rightarrow X$ of the form $T=y^{*} \otimes x$, where $x \in X$ and $y^{*} \in Y$. A Banach space $X$ is called an almost Daugavet space or a space with the almost Daugavet property 
if it has the Daugavet property with respect to some norming subspace $Y \subset X^{*}$. This definition, which was introduced in [1], is a generalization of the Daugavet property. Recall that a Banach space $X$ has the Daugavet property if it has the Daugavet property with respect to $X^{*}$.

In the following section we study some structural properties of the class of almost Daugavet spaces, and our main tool will be Theorem 1.1. We show that the $\ell_{1}$-sum and $\ell_{\infty}$-sum of separable almost Daugavet spaces are almost Daugavet spaces.

Concerning subspaces, we show results about $L$-summands and $M$-ideals. But the main result will be that the almost Daugavet property of a separable Banach space $X$ is inherited by a closed subspace $Z$ if the quotient space $X / Z$ contains no copy of $\ell_{1}$.

Special thanks go to V. Kadets for giving an important hint for achieving this result in such a general setting.

\section{Subspaces and sums of Almost Daugavet spaces}

2.1. Sums. The almost Daugavet property depends very much on the norm of a space. So it cannot be expected that arbitrary sums of almost Daugavet spaces still have the almost Daugavet property. But there are the following results concerning $\ell_{1}$ - and $\ell_{\infty}$-sums.

Proposition 2.1. If $X$ and $Y$ are two separable almost Daugavet spaces, then $X \oplus_{\infty} Y$ is an almost Daugavet space as well.

Proof. As a consequence of Theorem 1.1, $X$ as well as $Y$ contains a canonical $\ell_{1}$ type sequence $\left(e_{n}\right)_{n \in \mathbb{N}}$, resp. $\left(f_{n}\right)_{n \in \mathbb{N}}$. Every member of $\left(\left(e_{n}, f_{n}\right)\right)_{n \in \mathbb{N}}$ is an element of $B_{X \oplus_{\infty} Y}$ and for $(x, y) \in X \oplus_{\infty} Y$ we have

$$
\begin{aligned}
\lim _{n \rightarrow \infty}\left\|(x, y)+\left(e_{n}, f_{n}\right)\right\| & =\lim _{n \rightarrow \infty} \max \left\{\left\|x+e_{n}\right\|,\left\|y+f_{n}\right\|\right\} \\
& =\max \left\{\lim _{n \rightarrow \infty}\left\|x+e_{n}\right\|, \lim _{n \rightarrow \infty}\left\|y+f_{n}\right\|\right\} \\
& =\max \{\|x\|+1,\|y\|+1\} \\
& =\max \{\|x\|,\|y\|\}+1=\|(x, y)\|+1
\end{aligned}
$$

So $\left(\left(e_{n}, f_{n}\right)\right)_{n \in \mathbb{N}}$ is a canonical $\ell_{1}$-type sequence in $X \oplus_{\infty} Y$ and $X \oplus_{\infty} Y$ is an almost Daugavet space by Theorem 1.1.

Proposition 2.2. Let $X$ and $Y$ be separable Banach spaces. If $X$ has the almost Daugavet property, then so does $X \oplus_{1} Y$.

Proof. Because of Theorem 1.1, $X$ contains a canonical $\ell_{1}$-type sequence $\left(e_{n}\right)_{n \in \mathbb{N}}$. For $(x, y) \in X \oplus_{1} Y$ we get

$$
\begin{aligned}
\lim _{n \rightarrow \infty}\left\|(x, y)+\left(e_{n}, 0\right)\right\| & =\lim _{n \rightarrow \infty}\left(\left\|x+e_{n}\right\|+\|y\|\right)=\lim _{n \rightarrow \infty}\left\|x+e_{n}\right\|+\|y\| \\
& =\|x\|+\|y\|+1=\|(x, y)\|+1
\end{aligned}
$$

and thus $\left(\left(e_{n}, 0\right)\right)_{n \in \mathbb{N}}$ is a canonical $\ell_{1}$-type sequence. Therefore $X \oplus_{1} Y$ is an almost Daugavet space as a result of Theorem 1.1. 
2.2. Subspaces. Now we are interested in the question concerning which subspaces of almost Daugavet spaces inherit the almost Daugavet property. Since the almost Daugavet property depends very much on the norm of a space, we are going to study subspaces fulfilling adequate conditions.

Proposition 2.3. Let $X$ be a separable almost Daugavet space, let $J$ be an $L$ summand and let $\widehat{J}$ be the complementary L-summand (i.e., $X=J \oplus_{1} \widehat{J}$ ). Then $J$ or $\widehat{J}$ has the almost Daugavet property.

Proof. As a result of Theorem 1.1 it suffices to show that $T(J)=2$ or $T(\widehat{J})=2$.

Let us suppose that $T(J)<2$ and $T(\widehat{J})<2$. Then there exist for an $\varepsilon>0$ inner $(2-2 \varepsilon)$-nets of $S_{J}$ and $S_{\widehat{J}}$. Let $\left\{j_{1}, \ldots, j_{l}\right\}$ be such an inner $(2-2 \varepsilon)$-net of $S_{J}$ and let $\left\{\widehat{j}_{1}, \ldots, \widehat{j}_{m}\right\}$ be one of $S_{\widehat{J}}$. Furthermore, let $\left\{\lambda_{1}, \ldots, \lambda_{n}\right\}$ be an inner $(\varepsilon / 2)$-net of $[0,1]$.

As $J$ and $\widehat{J}$ are $L$-summands, the unit sphere of $X$ can be expressed by

$$
S_{X}=\left\{(\lambda j,(1-\lambda) \widehat{j}): \lambda \in[0,1], j \in S_{J}, \widehat{j} \in S_{\widehat{J}}\right\} .
$$

Given $x=(\lambda j,(1-\lambda) \widehat{j}) \in S_{X}$, we can pick $\lambda_{0} \in\left\{\lambda_{1}, \ldots, \lambda_{n}\right\}$ with $\left|\lambda_{0}-\lambda\right| \leq \varepsilon / 2$, $j_{0} \in\left\{j_{1}, \ldots, j_{l}\right\}$ with $\left\|j_{0}-j\right\| \leq 2-2 \varepsilon$, and $\widehat{j}_{0} \in\left\{\widehat{j}_{1}, \ldots, \widehat{j}_{m}\right\}$ with $\left\|\widehat{j_{0}}-\widehat{j}\right\| \leq 2-2 \varepsilon$. Then we get with $y=\left(\lambda_{0} j_{0},\left(1-\lambda_{0}\right) \widehat{j}_{0}\right)$ :

$$
\begin{aligned}
\|x-y\| & =\left\|\lambda j-\lambda_{0} j_{0}\right\|+\left\|(1-\lambda) \widehat{j}-\left(1-\lambda_{0}\right) \widehat{j_{0}}\right\| \\
& =\left\|\left(\lambda-\lambda_{0}\right) j+\lambda_{0}\left(j-j_{0}\right)\right\|+\left\|\left(\lambda_{0}-\lambda\right) \widehat{j}+\left(1-\lambda_{0}\right)\left(\widehat{j}-\widehat{j}_{0}\right)\right\| \\
& \leq\left|\lambda-\lambda_{0}\right|(\|j\|+\|\widehat{j}\|)+\left(\lambda_{0}\left\|j-j_{0}\right\|+\left(1-\lambda_{0}\right)\left\|\widehat{j}-\widehat{j}_{0}\right\|\right) \\
& \leq \varepsilon+(2-2 \varepsilon)=2-\varepsilon .
\end{aligned}
$$

Thus $\left\{(\lambda j,(1-\lambda) \widehat{j}): \lambda \in\left\{\lambda_{1}, \ldots, \lambda_{n}\right\}, j \in\left\{j_{1}, \ldots, j_{l}\right\}, \widehat{j} \in\left\{\widehat{j}_{1}, \ldots, \widehat{j}_{m}\right\}\right\}$ is an inner $(2-\varepsilon)$-net of $S_{X}$. But this is because of Theorem 1.1 a contradiction to the assumption that $X$ has the almost Daugavet property.

Recall that an $M$-ideal in a Banach space $X$ is a closed subspace $J$ such that $X^{*}$ decomposes as $X^{*}=Y \oplus_{1} J^{\perp}$ for some closed subspace $Y$ of $X^{*}$, where $J^{\perp}=\left\{x^{*} \in X^{*}: x^{*}(x)=0\right.$ for all $\left.x \in J\right\}$.

In [3, Proposition 2.10] it is shown that $M$-ideals of Banach spaces with the Daugavet property have the Daugavet property as well. The same proof works for almost Daugavet spaces.

Proposition 2.4. Let $X$ be a Banach space, let $Y$ be a norming subspace of $X^{*}$ and suppose that $X$ has the Daugavet property with respect to $Y$. If $J \neq\{0\}$ is an $M$-ideal in $X$, then $J$ has the Daugavet property with respect to $\left\{\left.y^{*}\right|_{J}: y^{*} \in Y\right\}$. So $J$ is an almost Daugavet space as well.

On the other hand, almost Daugavet spaces are in a certain sense "big". They have thickness 2 and contain a copy of $\ell_{1}$. So in the next theorem we consider "big" subspaces of an almost Daugavet space.

Theorem 2.5. Let $X$ be a separable almost Daugavet space and let $Z$ be a closed subspace of $X$. If the quotient space $X / Z$ contains no copy of $\ell_{1}$, then $Z$ has the almost Daugavet property too. 
Proof. By Theorem 1.1, it suffices to construct a canonical $\ell_{1}$-type sequence in $Z$.

Let $E_{1} \subset E_{2} \subset E_{3} \subset \ldots$ be a sequence of finite-dimensional subspaces whose union is dense in $X$. Also, fix a sequence of positive real numbers $\left(\varepsilon_{n}\right)_{n \in \mathbb{N}}$ that converges monotonically towards zero. As a consequence of Theorem 1.1 and Proposition 1.2. there is a canonical $\ell_{1}$-type sequence $\left(e_{n}\right)_{n \in \mathbb{N}}$ in $B_{X}$ satisfying the following condition: for every $x \in E_{n}$ and every $y=\sum_{k=n+1}^{M} \lambda_{k} e_{k}$ we have

$$
\|x+y\| \geq\left(1-\varepsilon_{n}\right)\|x\|+\sum_{k=n+1}^{M}\left(1-\varepsilon_{k-1}\right)\left|\lambda_{k}\right| .
$$

So the space $\overline{\operatorname{span}}\left\{e_{n}: n \geq k\right\}$ is isomorphic to $\ell_{1}$ for each $k \in \mathbb{N}$. Since $X / Z$ contains no copy of $\ell_{1}$, the quotient map $\pi: X \rightarrow X / Z$ fails to be bounded below on each subspace $\overline{\operatorname{span}}\left\{e_{n}: n \geq k\right\}$; i.e., for each $k \in \mathbb{N}$, there is no $C>0$ with $C\|x\| \leq\|\pi(x)\|$ for all $x \in \overline{\operatorname{span}}\left\{e_{n}: n \geq k\right\}$.

As a result of this, there is a linear combination $\sum_{k=1}^{m_{1}} \lambda_{k} e_{k}$ with $\left\|\sum_{k=1}^{m_{1}} \lambda_{k} e_{k}\right\|=$ 1 and $\left\|\pi\left(\sum_{k=1}^{m_{1}} \lambda_{k} e_{k}\right)\right\| \leq \varepsilon_{1} / 4$. By the definition of the quotient norm, there is an $\hat{f}_{1} \in Z$ with $\left\|\hat{f}_{1}-\sum_{k=1}^{m_{1}} \lambda_{k} e_{k}\right\| \leq \varepsilon_{1} / 2$, and we get $\left\|f_{1}-\sum_{k=1}^{m_{1}} \lambda_{k} e_{k}\right\| \leq \varepsilon_{1}$ with $f_{1}=\hat{f}_{1} /\left\|\hat{f}_{1}\right\|$. Going on like this, we get for each $n \in \mathbb{N}$ a linear combination $\sum_{k=m_{n-1}+1}^{m_{n}} \lambda_{k} e_{k}$ with $\left\|\sum_{k=m_{n-1}+1}^{m_{n}} \lambda_{k} e_{k}\right\|=1$ and an element $f_{n} \in S_{Z}$ with $\left\|f_{n}-\sum_{k=m_{n-1}+1}^{m_{n}} \lambda_{k} e_{k}\right\| \leq \varepsilon_{n}$.

It remains to show that $\left(f_{n}\right)_{n \in \mathbb{N}}$ is a canonical $\ell_{1}$-type sequence. Given $x \in X$ and $\varepsilon>0$, we can assume without loss of generality that $x \in E_{\hat{n}}$ for an $\hat{n} \in \mathbb{N}$, because the union of all subspaces $E_{n}$ is dense. The sequence $\left(\varepsilon_{n}\right)_{n \in \mathbb{N}}$ converges monotonically towards zero. So there is an $N_{0} \in \mathbb{N}$ with $\varepsilon_{n-1}\|x\|+\varepsilon_{n-1}+\varepsilon_{n} \leq \varepsilon$ for all $n>N_{0}$. Then we get for all $n>\max \left\{\hat{n}, N_{0}\right\}$ using (2.1) and $m_{n-1} \geq n-1$

$$
\begin{aligned}
\|x\|+1 \geq\left\|x+f_{n}\right\| & \geq\left\|x+\sum_{k=m_{n-1}+1}^{m_{n}} \lambda_{k} e_{k}\right\|-\left\|\sum_{k=m_{n-1}+1}^{m_{n}} \lambda_{k} e_{k}+f_{n}\right\| \\
& \geq\left(1-\varepsilon_{n-1}\right)\|x\|+\sum_{k=m_{n-1}+1}^{m_{n}}\left(1-\varepsilon_{k-1}\right)\left|\lambda_{k}\right|-\varepsilon_{n} \\
& \geq\left(1-\varepsilon_{n-1}\right)\|x\|+\left(1-\varepsilon_{n-1}\right) \sum_{k=m_{n-1}+1}^{m_{n}}\left|\lambda_{k}\right|-\varepsilon_{n} \\
& \geq\left(1-\varepsilon_{n-1}\right)\|x\|+\left(1-\varepsilon_{n-1}\right)-\varepsilon_{n} \\
& \geq\|x\|+1-\varepsilon .
\end{aligned}
$$

So $\lim _{n \rightarrow \infty}\left\|x+f_{n}\right\|=\|x\|+1$ and $\left(f_{n}\right)_{n \in \mathbb{N}}$ is a canonical $\ell_{1}$-type sequence.

\section{REFERENCES}

[1] V. Kadets, V. Shepelska, and D. Werner, Quotients of Banach spaces with the Daugavet property, Bull. Pol. Acad. Sci. 56 (2008), no. 2, 131-147. MR2431006 (2009f:46011)

[2] _ Thickness of the unit sphere, $\ell_{1}$-types, and the almost Daugavet property (2009), to appear in Houston J. Math., available at http://arxiv.org/abs/0902.4503v1.

[3] V. M. Kadets, R. V. Shvidkoy, G. G. Sirotkin, and D. Werner, Banach spaces with the Daugavet property, Trans. Amer. Math. Soc. 352 (2000), 855-873. MR1621757 (2000c:46023) 
[4] J.-L. Krivine and B. Maurey, Espaces de Banach stables, Israel J. Math. 39 (1981), 273-295. MR636897 (83a:46030)

[5] R. Whitley, The size of the unit sphere, Canadian J. Math 20 (1968), 450-455. MR0228997 $(37: 4576)$

Department of Mathematics, Freie Universität Berlin, Arnimallee 6, 14195 Berlin, GERMAnY

E-mail address: simon.luecking@fu-berlin.de 